\title{
La crosta terrestre, dagli Appennini all'Atlantico, ricostruita sulla base dei rilievi sismici (*)
}

\author{
PIETRO CALOI
}

Da oltre due decemi, a più riprese, la mia attenzione è stata portata sul problema delle caratteristiche fisiche della crosta terrestre, con particolare riguardo alle stratifificazioni di cui risulta costituita, in corrispondenza dei sistemi di montagne (Alpi, Appennini), degli altipiani e delle grandi valli (Schwäbische Alb, Valle del Po, ecc.).

Prima di riassumere i risultati delle indagini, condotte nel senso sopra detto, da me e da miei collaboratori, ritengo opportuno aceemnare rapidamente ai metodi di calcolo di cui, via via, si è fatto uso.

1. - Nella fase iniziale delle mie ricerche mi sono valso - come ela naturale - dei procedimenti di calcolo allora più in uso.

Per la determinazione delle coordinate ipocentrali godeva molto farore il metodo di Inglada, mentre per la determinazione del solo epicentro er:a molto diffuso il metodo delle iperboli di $\Lambda$. Molıorovicic. Tali metodi - oggi in parte superati - somo troppo noti perchè io debba riassumerli.

Per una prima approssimazione che, nel aso di terremoti decisamente superficiali poteva ritenersi senz'altro arcettabile - mi sono servito, per qualche anno, di metodi grafici, basati sulla greometria dei contatti $\left({ }^{4}\right)$.

Supponiamo di avere a disposizione i tempi d'inizio delle onde $P g$ in tre strazioni diverse $O_{1}, O_{2}, O_{3}$. Indichiamo questi tempi con $t_{1}, t_{2}, t_{3}$ e poniamo $\delta_{1}=t_{2}-t_{1}, \delta_{2}=t_{3}-t_{1}$.

Il metodo si ricondure alla ricerca del centro della circonferenza passante per un

(*) Per grentile concessione del Prof. B. Brockamp, questa Nota - gia pubblicata sul n. 2 del 1958 dello "Zeitschrift fur Geopliysik" - viene ora pubblicata in lingua italiana. punto (la stazione fondamentale $O_{1}$ ) e tangente a due circonferenze date (avanti centro nelle stazioni $\mathrm{O}_{2}, \mathrm{O}_{3}$ e raggi uguali a $v_{p g} \cdot \delta_{1}$ e $v_{l^{\prime}} \cdot \delta_{2}$ rispettivamente). Le cantele per l'applicazione del metodo, la risoluzione del problema e le soluzioni possibili sono discusse nelle memorie originali, alle quali rimando il lettore $\left({ }^{4,5,6}\right)$.

Risolsi pure geometricamente il caso di quattro stazioni, una delle quali, la più vicina, scelta come fondamentale. Conosciamo i tempi di registrazione delle onde $P g(o S g)$ nelle stazioni date $O_{1}, O_{2}, O_{3}, O_{4}$. Consideriamo le differenze $\delta_{1}, \delta_{2}, \delta_{3}$ fra $\mathrm{i}$ tempi di registrazione delle $P g$ nelle tre stazioni più lontane e il tempo delle $P g$ nella stazione fondamentale. Le tre circonferenze di mggi $v_{P g} \cdot \delta_{1}, v_{P g} \cdot \delta_{2}, v_{P g} \cdot \delta_{3}$ e centri il $O_{1} . O_{2}, O_{3}$ dovramno risultare tangenti ad una circonferenza che ha per centro l'epicentro. Il problema si riduce quindi alla ricerca del centro della circonferenza tangente esternamente a tre circonferenze date.

Diodi successivamente forma amalitica a quest'ultimo caso (1').

Indichiamo con $\left(o_{2}\right),\left(o_{3}\right),\left(o_{4}\right)$ le tre circonferenze assegnate di raggi $r, r^{\prime}, r^{\prime \prime}$ rispettivamente, e con $(E)$ la circonferenza incognita di laggio $R$. Poichè $(E)$ deve risultare tangente a $\left(O_{2}\right),\left(o_{3}\right),\left(o_{1}\right)$ le coordinate del suo centro soddisfano alle relazioni

$$
\begin{aligned}
& O_{2}=R(R+2 r) ; \\
& O_{3}=R\left(R+2 r^{\prime}\right) ; \\
& O_{4}=R\left(R+2 r^{\prime \prime}\right),
\end{aligned}
$$

dove con $O_{2}, O_{3}, O_{4}$ si indicano le equazioni delle circonferenze date. 
Poniamo l:origine delle coordinate al centro della circonferenza $\left(\sigma_{2}\right)$ e indichiamo con $x$, y le coordinate ded punto di contatto di $\left(O_{2}\right)$ con $(E)$. Se $Y, Y$ sono le coordinate del centro di $(E)$, si ha manifestamente

$$
Y=\frac{r(R+r)}{r}, \quad Y=\frac{y(R+r)}{r} .
$$

$I, Y$ devomo inoltre reriticale le equazioni

$$
\begin{aligned}
& O_{2}-O_{3}=2 R\left(r-r^{\prime}\right) \\
& O_{2}-O_{3}=2 R\left(r-r^{\prime \prime}\right) .
\end{aligned}
$$

Indichiamo con $a^{\prime}, \beta^{\prime}$; $a^{\prime \prime}, \beta^{\prime \prime}$ le coordinate dei centri di $\left(O_{3}\right),\left(O_{4}\right)$ rispetto all'origine [centro di $\left.\left(o_{2}\right)\right]$. La sostituzione di $X, Y$ a $x, y$ nelle (2), tenemdo conto delle equazioni di $\left(o_{2}\right),\left(o_{3}\right),(o, 4)$ messe sotto forma genelale, ronduce alle relazioni

$$
\begin{aligned}
& (R+r)\left(O_{2}-O_{3}\right)=R\left[\left(r-r^{\prime}\right)^{2}-\alpha^{\prime 2}-\beta^{\prime 2}\right] \\
& (R+r)\left(O_{2}-O_{4}\right)=R\left[\left(r-r^{\prime \prime}\right)^{2}-\alpha^{\prime 2}-\beta^{\prime \prime 2}\right] .
\end{aligned}
$$

Consegue che il punto di contatto cereato si trova all'intersezione della circonferenza $\left(o_{2}\right)$ con la retta

$$
\frac{O_{2}-O_{3}}{a^{\prime 2}+\beta^{\prime 2}-\left(r-r^{\prime}\right)^{2}}=\frac{\left(O_{2}-()_{1}\right.}{a^{\prime \prime 2}+\beta^{\prime \prime 2}-\left(r-r^{\prime \prime}\right)^{2}},
$$

che, in forma esplicita, può scriversi

$$
\begin{aligned}
& \frac{a^{\prime} x+\beta^{\prime} y+\left(r^{\prime}-r\right) r}{a^{\prime 2}+\beta^{\prime 2}-\left(r-r^{\prime}\right)^{2}}= \\
= & \frac{a^{\prime \prime} x+\beta^{\prime \prime} y+\left(r^{\prime \prime}-r\right) r}{a^{\prime \prime 2}+\beta^{\prime \prime 2}-\left(r-r^{\prime \prime}\right)^{2}} .
\end{aligned}
$$

Poichè

$$
O_{2}=x^{2}+y^{2}-r^{2}=0,
$$

fatte le posizioni

$$
\begin{gathered}
Q^{\prime 2}=a^{\prime 2}+\beta^{\prime 2}-\left(r-r^{\prime}\right)^{2} ; \\
\varrho^{\prime \prime 2}=\iota^{\prime \prime 2}+\beta^{\prime \prime 2}-\left(r-r^{\prime \prime}\right)^{2}, \\
\varepsilon_{1}=Q^{\prime \prime 2} r^{\prime}-r^{\prime 2} a^{\prime \prime} \quad ; \quad \varepsilon_{2}=\varrho^{\prime \prime 2} \beta^{\prime}-\varrho^{\prime 2} \beta^{\prime \prime}, \\
\sigma=\varrho^{\prime 2}\left(r^{\prime \prime}-r\right)-Q^{\prime \prime 2}\left(r^{\prime}-r\right), \\
x=1 \varepsilon_{1}^{2}+\varepsilon_{2}^{2}-\sigma^{2},
\end{gathered}
$$

dalle $[4],[5]$ consegue

$$
x=r \frac{\sigma \varepsilon_{1} \mp \varkappa \varepsilon_{0}}{\varepsilon_{1}^{2}+\varepsilon_{2}^{2}}, y=r \frac{\sigma \varepsilon_{2} \pm \chi \varepsilon,}{\varepsilon_{1}^{2}+\varepsilon_{2}^{2}} .
$$

D)altronde, dalle [3] si ha

$$
\begin{array}{r}
R=\frac{r}{4}\left[\frac{r^{2}-r^{\prime 2}+\alpha^{\prime 2}+\beta^{\prime 2}-2 \alpha^{\prime} x-2 \beta^{\prime} y}{\alpha^{\prime} x+\beta^{\prime} y+r\left(r^{\prime}-r\right)}+\right. \\
\left.+\frac{r^{2}-r^{\prime \prime 2}+\beta^{\prime \prime 2}-2 \alpha^{\prime \prime} x-2 \beta^{\prime \prime} y}{\alpha^{\prime \prime} x+\beta^{\prime \prime} y+r\left(r^{\prime \prime}-r\right)}\right] .
\end{array}
$$

Sostituite le [6] nella [7], si ottengono due valori per $R$. Le [1] damno allora due coppie di valori per $X, Y$. Si vede immediatamente quale di queste coppie costituisce le coordinate dell'epicentro. L'altra coppia dà le coordinate dell'epicentro. L'alt ra coppia da le coordinate del rentro della circonferenza che tange le tre cinconferenze date nei tre punti più discosti dal dentro radicale. La [7] dà per il raggio di quest'ultima circonferenza un valore positivo solo quando essa non comprende le circonferenze assegnate.

2. - Quasi tutti i metorli, analitici o grafici, per la determinazione delle coordinate spaziali di un terremoto sfruttano eschusivamente i tempi di registrazione delle onde longitudinali dirette (terremoti vicini) o rifratte (terremoti lontani).

Il motivo principale di questa preferenza deve ricercarsi nel fatto whe le onde $P g$ e $P_{n}$ iniziano un sisnogramma e pertanto i tempi che ad esse si riferiscono, nel caso di inizi chiari, possono essere determinati con molta precisione. Ugual precisione nom sempre è possibile nei tempi che si riferiscono all'inizio delle onde trasversali $(S g$ o $S n$ ), in quanto queste onde rengono registrate nel corso del sismoglamma e il loro inizio può apparire incerto. In effetti però è da osservare che, se questo puo talvolta aceadere, in compenso le onde sig a sn sono generalmente molto più ampie delle $P g$ e $P_{n}$ e che gli inizi ad impeto sono facimente individuabili. Studi accumati compiuti negli ultimi recenni su terremoti ad origine vicina, hamo provato (he le onde $s g$ sono individuabili entro liniti d'errore spesso piu picooli di quelli relativi alle onde $\mathrm{Pg}$.

I metodi che famno uso dei tempi riferentisi alle onde longitudinali possono quindi estendersi senz'altro ai tempi di inizio delle onde trassererali.

Poichè interpretazioni aceurate possono consentire uguale fiducia ai tempi di regi- 
strazione delle onde longitudinali e trassersali. un metolo che sfruttasse contemporaneamente i tempi deinizio dei doe diversi tipi di onde, presenterebbe sugli altri un notevole vantaggio: quello di lasciare i risultati innmuni dagli errori di correzione del tempo, errori che, in molte stazioni ancora, somo tutt'alt ro che trascourabili $\left({ }^{8}\right)$. Simile motodo può anzi prescinclere dai tempi corretti a, guando si conosea con precisione la relocità di scorrimento dei rulli registratori, puó perpettere persino l'uso di sismogrammi privi dei segnali orari $\left(^{8}\right)$.

Indichiamo con $t_{2}$ a $t_{2}$ i tempi di registrazione delle onde Pg ad sig in una stazione sismica di distanza epicentrale A. Se indichiamo con $\Delta_{h}$ la distanza ipocentro-stazione sismical, e con $v_{1}$ e $v_{2}$ le velocità di propagazione delle onde Pg ed sig rispettivamente, Sillì:

$$
\Delta_{h}=\frac{v_{1} \cdot v_{2}}{v_{1}-v_{2}}\left(t_{2}-t_{1}\right) .
$$

Fanciamo la posizione:

$$
k=\frac{v_{1} \cdot v_{2}}{v_{1}-v_{2}} .
$$

Consideriamo le tre sfere di centri 1, 2, 3 e raggi $k T_{1}, k T_{2}$ e $k T_{3}$ rispettivamente. Riferiamori ad un sistema di assi ortogonali facendo coincidere la superficie terrester, supposta piana, con il piano $x y$ : siano $x_{0}$, $y_{0} ; x_{1} ; y_{1} ; y_{2}, e y_{2}, x_{3}, y_{3}$ le coordinate dellepicentro e delle tre stazioni 1, 2, 3. Conseguono le equazioni:

$$
\begin{gathered}
\left(x_{1}-x_{0}\right)^{2}+\left(y_{1}-y_{0}\right)^{2}+z_{0}{ }^{2}-\left(k T_{1}\right)^{2}=0 \\
\left(x_{2}-x_{0}\right)^{2}+\left(y_{2}-y_{0}\right)^{2}+z_{0}{ }^{2}-\left(k T_{2}\right)^{2}=0 \\
\left(x_{3}-x_{0}\right)^{2}+\left(y_{3}-y_{0}\right)^{2}+z_{0}{ }^{2}-\left(k T_{3}\right)^{2}=0 \\
T_{i}=\left(t_{2}-t_{1}\right) i \quad, \quad i=1,2,3 .
\end{gathered}
$$

Poniamo:

$$
\begin{aligned}
& 2 c_{1}=-\left(k T_{1}\right)^{2}+x_{1}{ }^{2}+y_{1}{ }^{2} \\
& 2 c_{2}=-\left(k T_{2}\right)^{2}+x_{2}{ }^{2}+y_{2}{ }^{2} \\
& 2 c_{3}=-\left(k \cdot T_{3}\right)^{2}+x_{3}{ }^{2}+y_{3}{ }^{2}
\end{aligned}
$$

ed eliminiamo $z_{0}$. Sottratendo, per esempio, la 20 o la $3^{\circ}$ delle [8] rispettivamente, dalla to si hamno le equazioni:

$$
\begin{aligned}
& x_{0}\left(x_{2}-x_{1}\right)+y_{0}\left(y_{2}-y_{1}\right)-\left(c_{2}-c_{1}\right)=0 \\
& x_{0}\left(x_{3}-x_{1}\right)+y_{0}\left(y_{3}-y_{1}\right)-\left(c_{3}-c_{1}\right)=0,
\end{aligned}
$$

che rappresentano gli assi malicali delle (oop)pie di circonferenze determinate dall'intersezione delle sfere 1-2, 1-3 con la superdicie terrestres.

Se ne dedurono, per le incognite, le espressioni:

$$
\begin{aligned}
& x_{0}=\frac{c_{1}\left(y_{2}-y_{3}\right)+c_{2}\left(y_{3}-y_{1}\right)+c_{3}\left(y_{1}-y_{2}\right)}{x_{1}\left(y_{2}-y_{3}\right)+x_{2}\left(y_{3}-y_{1}\left(+x_{3}\left(y_{1}-y_{2}\right)\right.\right.} \\
& y_{0}=\frac{c_{1}\left(x_{3}-x_{2}\right)+c_{2}\left(x_{1}-x_{3}\right)+c_{3}\left(x_{2}-y_{0}\right)}{x_{1}\left(y_{2}-y_{3}\right)+x_{2}\left(y_{3}-y_{1}\right)+x_{3}\left(y_{1}-y_{2}\right)} .
\end{aligned} .
$$

Potendo disporre dei dati di quattro o più stazioni, il problema può essere risolto rol metodo dei minimi quadlati.

Sia $i$ il numero delle stazioni. Se riferiamo tutte le stazioni ald una (la prima) sceelta come fondamentale, le equazioni degli assi ladicali conducono al sistema:

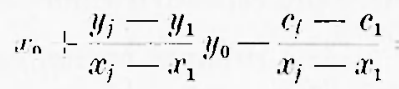

$$
\begin{aligned}
& (j=2,3, \ldots n)
\end{aligned}
$$

essemento:

$$
\begin{gathered}
2 c_{i}=-\left(k_{i} T^{\prime}{ }_{i}\right)^{2}+x_{i}{ }^{2}+y_{i}{ }^{2},(i=1,2,3, \ldots n) \\
T_{i}=\left(t_{2}-t_{1}\right) i,
\end{gathered}
$$

dove $t_{1}$ a $t_{2}$ sono $\mathrm{i}$ tempi di registrazione delle onde $P g$ ed $\mathrm{Sg}$ rispettivamente, in una stessa stazione.

Poniamo:

$$
u=\frac{u_{i}-u_{1}}{x_{i}-x_{1}} ; \beta=\frac{c_{j}-c_{1}}{x_{i}-x_{1}}(j=2,3, \ldots u) .
$$

Ie [9] si riducono a:

$$
x_{0}+\alpha y_{0}=\beta .
$$

To equazioni normali sono pertanto:

$$
\begin{aligned}
& {[1 \cdot 1] x_{0}+[1 \cdot \alpha] y_{0}=[1 \cdot \beta]} \\
& {[1 \cdot \alpha] x_{0}+[\alpha \cdot \alpha] y_{0}=[\alpha \cdot \beta],}
\end{aligned}
$$

da cui:

$$
\begin{aligned}
& y_{0}=\frac{[\alpha \beta \cdot 1]}{[\alpha \alpha \cdot 1]} ; \\
& x_{0}=-\frac{[1 \cdot \alpha]}{[1 \cdot 1]}, \frac{[1 \cdot \beta]}{[1 \cdot 1]},
\end{aligned}
$$


dove:

$$
\begin{aligned}
& {[\alpha \beta \cdot 1]=[\alpha \beta]-\frac{[1 \cdot \alpha]}{[1 \cdot 1]}[1 \cdot \beta]:} \\
& {[\alpha \alpha \cdot 1]=[\alpha \cdot \alpha]-\frac{[1 \cdot \alpha]}{[1 \cdot i]}[1 \cdot \alpha] .}
\end{aligned}
$$

Se invere consideriamo le stazioni tutte alla stessa stregua, il sistema da risolvere diventa:

$$
\begin{gathered}
x_{0}+\frac{y_{j}-y_{j-1}}{x_{j}-x_{j-1}} y_{n}-\frac{x_{j}-x_{j-1}}{c_{j}-c_{1-1}}=0 \\
(j=2,3, \ldots n)
\end{gathered}
$$

e valgono ancora le [11], salvo che in esse va fatto:

$$
\begin{gathered}
\iota=\frac{y_{j}-y_{j-1}}{x_{j}-x_{j-1}}: \rho=\frac{c_{j}-c_{j-1}}{x_{j}-x_{j-1}} \\
\quad(j=2,3, \ldots n) .
\end{gathered}
$$

Nel metorlo sopra esposto il valore del rapporto $k=\frac{v_{1} \cdot v_{2}}{v_{1}-v_{2}}$ i ritenuto costante, il che equivale a considerare costante il valore della velocità delle onde longitudinali e trasversali dirette, cosa generalmente sufficiente ai fini pratici.

In una nota successiva $\left({ }^{9}\right)$, ho ritemuto opportuno considerare incognite le granzezze $v_{1}, v_{2}$; oltre a consentire una maggiore precisione noi calcoli, cio avrebbe permesso, applicando il metorlo a numerosi terremoti, di ottenere la legge di variazione della velocità in funzione della profondità.

Espongo per sommi (appi i principi su cui a basato il nuovo metorlo accemato.

Indichiamo oon $t_{1}, t_{2}$ i tempi di registrazione delle onde $P g, S g$ in una stazione assegnata; siano inoltre $x_{0}, y_{0}, z_{0} ; x_{i}, y_{i}, z_{i}$ rispettivamente le coordinate sparziali di un ipocentro a di una generica stazione di osservazione $S_{i}$, riferita alla medesina origine; il piano $x y$ coincirla con la superficie della Terra, ritenuta piana. La coordinata $z_{i}$, che esprime l'altezza della stazione sul livello del mare, ì trascurabile rispetto al valore delle altre glandezze in ginoco; faremo pertanto $z_{i}=0$.

Poniamo

$$
\begin{aligned}
\varphi_{j}= & \frac{1}{k} \sqrt{\left(x_{i}-x_{0}\right)^{2}+\left(y_{i}-y_{0}\right)^{2}} \overline{+z_{0}^{2}-} \\
& -\left(t_{2}-t_{1}\right)_{i}, \quad(\imath=1,2, \ldots n) .
\end{aligned}
$$

Jovrà essere

$$
\varphi_{i}=\varphi_{i}\left(x_{0}, y_{0}, z_{0}, l_{i}\right)=0 .
$$

Sia $i>4$. Indichiamo con $\left(x_{0}\right),\left(y_{0}\right),\left(z_{0}\right)$. (k) un sistema di valori approssimati per le incognite e poniamo:

$$
\begin{gathered}
x_{0}=\left(x_{0}\right)+x ; y_{0}=\left(y_{0}\right)+y ; z_{0}=\left(z_{0}\right)+ \\
+r ; k=\left(l_{i}\right)+k_{1},
\end{gathered}
$$

dove $x, y, z, k$, rappresentano le correzioni incognite.

Avremo, limitando lo sviluppo al primo termine:

$$
\begin{aligned}
p_{i}=\phi_{i}(o) & +\frac{\partial \varphi_{i}(o)}{\partial\left(x_{0}\right)} x+\frac{\partial m_{i}(o)}{\partial\left(y_{0}\right)} \\
y & +\frac{\partial \varphi_{1}(o)}{\partial\left(z_{0}\right)} z+\frac{\partial \varphi_{i}(o)}{\partial(k)} k_{1},
\end{aligned}
$$

dove

$$
\begin{aligned}
& \frac{\partial \varphi_{i}(o)}{\partial\left(x_{0}\right)}= \\
& -\frac{1}{(k)} \frac{\left(x_{0}\right)-x_{i}}{\sqrt{\left(x_{i}-\left(x_{0}\right)\right)^{2}+\left(y_{i}-\left(y_{0}\right)\right)^{2}+\left(z_{0}\right)^{2}}}=a_{i} \\
& \frac{\partial \varphi_{i}(o)}{\partial\left(y_{0}\right)}= \\
& =\frac{1}{(k)} \frac{\left(y_{0}\right)-y_{1}}{\sqrt{\left(x_{i}-\left(x_{0}\right)\right)^{2}+\left(y_{i}--\left(y_{0}\right)\right)^{2}+\left(z_{0}\right)^{2}}}=b_{i}
\end{aligned}
$$

$\partial p_{i}(o)$

$\partial\left(z_{0}\right)$

$$
=\frac{1}{\left(l_{i}\right)} \frac{\left(z_{0}\right)}{\sqrt{\left(x_{i}-\left(x_{0}\right)\right)^{2}+\left(y_{1}-\left(y_{0}\right)\right)^{2}+\left(z_{0}\right)^{2}}}=c_{i}
$$

$\partial \varphi_{1}(o)$

$\partial(k)$

$$
\begin{gathered}
=-\frac{1}{\left(k^{2}\right)} \sqrt{\left(x_{i}-\left(x_{0}\right)\right)^{2}+\left(y_{i}-\left(y_{0}\right)\right)^{2}+\left(z_{0}\right)^{2}}=\lambda_{i} \\
\varphi_{i}(o)=\frac{1}{(n)} \sqrt{\left(x_{i}-\left(x_{0}\right)\right)^{2}+\left(y_{i}-\left(y_{0}\right)\right)^{2}+\left(z_{0}\right)^{2}}- \\
-\left(t_{2}-t_{1}\right)_{i}=i \\
(i=1,2,3, \ldots n)
\end{gathered}
$$

Le equazioni da risolvere divengono quincli:

$$
\begin{gathered}
a_{i} x+b_{i} y+c_{i} z+d_{i} k_{1}+l_{i}=v_{\iota}, \\
(i=1,2,3, \ldots n)
\end{gathered}
$$

in cui i secondi membri rappresentano i residui dovuti agli error'i d'osservazione. 
Il verificarsi della condizione di minimo per la somma dei quadrati dei residui funzione di $x, y, z, h_{1}$ - conduce al sistema di equazioni normali, sotto la forma loro assegenata dat Gauss:

$$
\begin{aligned}
& {[a a] x+[a b] y+[a c] z+[a d] l_{1}+[a l]=0} \\
& {[a b] x+[b b] y+[b c] z+[b d] l_{1}+[b b]=0} \\
& [a c] x+[b c] y+[c c] z+[c d]] k_{1}+[c l]=0 \\
& {[a d] x+[b d] ! y+[c d] z+[d d l] l_{1}+[d l]=0}
\end{aligned}
$$

La soluzione del sistema di equazioni normali conduce alle expazioni finali:

$$
\begin{aligned}
& {[a d] x+[a b] y+[a c] z+[a d] k_{1}+[a l]=0} \\
& {[b b \cdot 1] y+[b c \cdot 1] z+[b d \cdot 1] k_{1}+[b l \cdot 1]=0} \\
& {[c c \cdot 2] z+[c d \cdot 2] k_{1}+[c l \cdot 2]=0} \\
& {[d d \cdot \cdot 3] k_{1}+[d l \cdot 3]=0}
\end{aligned}
$$

con evidente significato dei simboli [bb.1], . $\ldots$. [ll .3].

E bene calcolate, comer verifien, anche lo schema [ll.4], in quanto esso, come è noto dalla teoria degoli errori, se i calcoli sono esalti, deve uguagliare la somma dei quadrati degli errori $[v v]$.

Indirando con $\varepsilon, m_{x}, m_{y}, m_{n}, m_{k_{2}}$ rispettivamente l'errore medio dell'unità di peso e gli errori medi dei valori più probabili delle incognite, si hal:

$$
\begin{aligned}
& \varepsilon= \pm \sqrt{\frac{[l l \cdot 4]}{n-4}} ; \\
& m_{x}=\frac{\varepsilon}{1[a a \cdot 3]} ; \quad m_{v}=\frac{\varepsilon}{1[b b \cdot 3]} \text {; } \\
& m_{z}=\frac{\varepsilon}{1[c c \cdot 3]}: \quad m_{k_{1}}=\frac{\varepsilon}{1[d d \cdot 3]} .
\end{aligned}
$$

Per determinare [aa .3], [bb.3], [cc.3], basta ordinare i sistemi di equazioni normali partemblo rispettivamente dalle equazioni:

$$
\begin{gathered}
d_{i} k_{i}+c_{i} z+b_{i} y+a_{i} x=v_{i} \\
d_{i} k_{i}+c_{i} z+a_{i} x+b_{i} x+b_{i} y=v_{i} \\
a_{i} x+b_{i} y+d_{i} k_{1}+c_{i} z=v_{i} \\
\quad(i=1,2,3, \ldots l)
\end{gathered}
$$

Nel'applicazione del metolo esposto è necessario conosecere le coordinate ortogonali delle stazioni d'osservasione a delleppicentro provvisorio, rispetto ad un punto della superficie terrestre, scelto come origine.
3. - Per un controllo della profondità ipocentrale, una volta determinata tale grandezzar con metodi che danno contemporaneamente le coordinate epicent rali, o semplicemente per arere un valore applossimato di essa, ci si può valere di diversi metodi di alcolo.

Areemneró all un paio, da me introdotti in sismologia. Uno si basa sulla conoscenza dell'angolo d'emergenza delle onde P'g.

Nom starò ade esporre la teoria del metodo. Dirò solo che, oltre ad un'acenrata determinazione delle costanti strumentali, lil sua applicazione richiede la conoseenza della distanza epicentrale. Lat precisione del metodo è legata inoltre alla conoscenza, esatta fin quanto è possibile, del rapporto delle velocita delle onde Py es sif. Teoricamente, gli angoli d'emergenza si deteminano ron la formula:

$$
\begin{aligned}
& \cos ^{-} c= \\
& =\frac{1}{4+\frac{\left(1-\varrho_{1}\right)^{2}}{o_{1} \sin ^{2} \vartheta}}, 1+o_{1}-c\left(1-o_{1}+2 o_{1} \sin ^{2} \vartheta\right)+ \\
& \left.+2 \mid \underline{o}_{1} / 1-c\left(1-\theta_{1}\right)-c^{2} \theta_{1} \sin ^{2} \theta\right) \cdot \cos \theta_{1}^{\prime} .
\end{aligned}
$$

dove $\varrho_{1}=\left(1-\frac{1}{r_{0}}\right)^{2}$, h è la profondità ipocentrale, 9 la distanza epicentrale in misura angolare, $r_{0}$ il raggio della Terra a $c$ è ma costante che, nel caso dell'Europa centrale, assume per le $P y$ il valie $c=7,76582$.

Nella mia ultima nota sullargomenti $\left({ }^{8}\right)$. sono riportati i valori di e per profondita da 1 a $50 \mathrm{~K}$ Km e pef clistanze epicentrali da 10 a $550 \mathrm{Km}$.

Com'e noto, dai sismogrammi si trae l'angolo d'emerengenza apparente e com la semplice formula:

$$
\operatorname{tang} e=\frac{x_{Z}}{\sqrt{x_{N}^{2}+x_{E}^{2}}},
$$

essendo $x_{Z}, x_{N}, x_{E}$ gli spostamenti reali del suolo in senso verticale e nelle direzioni orizzontali N-S, E-W rispettivamente.

Noto e, si risale poi all'angolo vero con la formula:

$$
\cos \bar{e}=\frac{v_{P g}}{v_{S g}} \sqrt{\frac{1}{2}(1-\sin \bar{e})} .
$$


Determinato e e nota la ristanzal epicentrale, dalla tabella da me calcolata si dedure subito il valore di $h$.

I metodi basati sull'angolo d'encrgenza delle onde longitudinali dirette presentano, sui precedenti, il vantaggio di essere indlipendenti dagli eventuali errori dei tempi d'osservazione. In questo caso però si esigono costanti strumentali determinate con la massima accuratezza ed impeti netti ed ampli della fase che serve allo scopo. Naturalmente, anche per questi metodi si presenta l'incertezza dovuta alla deviazione, più o meno mareata, del laggerio sismiro sotto l'azione degli strati superiori. Questa azione è del tutto trascarabile nel caso di stazioni situate su stratificazioni antiche e profonde. Sono cla evitare, in genere, i clati forniti cla stazoni poggianti su strati nolto spessi di terreni reeenti, alluvionali.

Un altro metodo da me irleato si fonda su noti principi alella teoria dei contatti.

$S_{e} S_{1}, C$ sono due stazioni (che poi possiamo pensare contenute nello stesso piano verticale per l'epicentro) di distanze epi(entrali note $A_{1}, A_{2}$ (*on $\left.A_{2}>A_{1}\right)$, se $t_{1}, t_{2}$ sono i tempi di registrazione delle $P y$ in $\aleph_{1}^{\prime}$, $C$, per profondità non nulle è sempre $\left(t_{2}-\right.$ $\left.t_{1}\right) v_{P g}<A_{2}-A_{1}$. Conoseendo la differenza $\left(A_{2}-A_{1}\right)-\left(t_{2}-t_{1}\right) t_{p g}$, abbiamo la possibiliti di dedurre facilmente la profondita delliporentro.

Con centro in $C$, trarediamo mal circonferenza di raggio uguale a $\left(t_{2}-t_{1}\right) v_{p g}$. Il problema si riduce a trofare il centro della circonferenza passante per i punti $S_{1}, S_{2}$, simmetrici rispetto allo epicentro, o tangente alla circenferenza traceiata. La lunghezza rlel segmento che misce questo centro (ipocentro) allepicentro è la profondità ipocentrica perseguita $\left.{ }^{5,6}\right)$.

Allo scopo di rendere il metodo più preciso (quando infatti il raggio $\left(t_{2}-t_{1}\right) v_{P^{2} g}$ è piuttosto grande, nella costruzione graficar la deterninazione del punto di tangente delle due circonferenze richiede nna rerta cantela), ho ritenuto opportuno dargli forma amaliticas.

Riferiano i punti del piano verticale per l'epicentro e le stazioni d'osservazione ad un sistema rli assi ortogonali, con l'asse $x$ pandellelo alla $C E$ (fig. 1), diretto positivamente dat $C$ verso $E$, e l'asse $y$ diretto positiva- mente verso l'interno della Terra (è sottinteso che, trattandosi di terremoti ad origine vicina, la siperficie terrestre, nei rlintorni dellepicentro, è ritenuta piana).

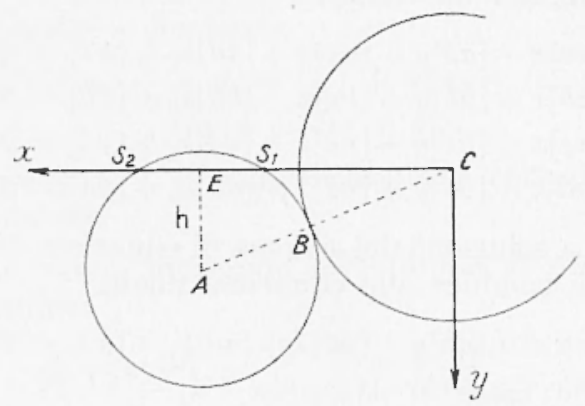

Fig. 1.

Siano $\left(x_{1}, y_{1}\right),\left(x_{2}, y_{2}\right)$ le cootrdinate dei punti $s_{1}, s_{2} ;$ poniamo $r_{2}=\left(t_{2}-t_{1}\right) v_{p g}$. Le incognite del problema sono le coordinate $\xi$, $\eta$ del punto $A$, le coordinate $x$, y del punto $B$ e il lagggio $r_{1}$ della rirconferenza di centro $A$, che passa per $S_{1}, S_{2}$ e tange in $B$ la circonferenzal di raggio $r_{2}$.

Poniamo $\gamma=\xi^{2}+\eta^{2}-r_{1}^{2}$; potreno consirlerare $\gamma$ in luogo di $\gamma_{1}$. Se poniamoin $C$ la origine degli assi coordinati e faceiamo coincidere la $x$ con la retta $C E$, conservando pere le $x, y$ il verso positivo prefisso, risolvendo il sistema a cui le incognite devono sodrlisfare, si perviene alla relazione

$$
y=h=1\left(\frac{r^{2}}{r}\right)^{2}-1 \cdot \frac{1}{2}\left(x_{1}+x_{2}\right),
$$

rove

$$
x=\frac{r_{2}^{2} \cdot\left(x_{1}+x_{2}\right)}{r_{2}^{2}+x_{1} \cdot x_{2}} .
$$

1. - Quando si hamo a disposizione registrazioni di stazioni lontane, allora può essere opportuno determinare le coordinate ipocentrali con metorli che si valgano dei tempi di tragitto delle onde $P n$.

Fu da me ideato, a questo scopo, 12 procedimento di adeolo che consente la contemporanea valutazione delle coordinate ipocentrali e del tempo origine $\left({ }^{46}\right)$.

Se indichiamo con $t_{0}, \lambda_{0}, \varphi_{0}$ e $h_{0}$ i valori approssimati delle incognite, con $\delta t_{0}, \delta \lambda_{0} e^{+}$ $\delta h_{0}$ le piecole correzioni ra alcolare con il metodo dei minini quadrati, per ottenere 
il valore più probabile delle incognite stesse e se $t_{n}$ rappresental il tempo di propagazione delle onte $P_{n}$, limitanto lo sviluppo in serie ai termini di primo ordine in $\delta / \mu_{0}, \delta \varphi_{0}$ es $\delta h_{0}$, supposti sufficientemente piccoli, si ha:

$t_{n}=f\left(\lambda_{0}, \varphi_{0}, h_{0}\right)+\frac{\partial f}{\partial \lambda_{0}} \delta \lambda_{0}+\frac{\partial f}{\partial \varphi_{0}}-\lambda m_{v}-\frac{\partial f}{\partial h_{0}} \delta I_{0}$.

In quest'ultima formula $f\left(\lambda_{0}, \tau_{v}, h_{0}\right)$ è il tempo di propagazione delle Pn corrispondenti allat distanzal epicentrale della stazione considerata, quale risulta dai valori approssimati di $\lambda_{0}, \varphi_{0}$; mentre $\frac{\partial f}{\partial h_{0}}$ si ottiene dalle dromo(rone, ritenemilo costante 1 nel valore 1 e assegllando ad $h$ (a partire $\left(h_{a} h_{0}\right.$ ) 101 incremento parli all'unità della dromocroma presceelta che darà il corrispondente incremento positivo o negativo del tempo di tragitto.

Si provid che:

$$
\begin{gathered}
\frac{\partial f}{\partial \lambda_{0}}=\frac{\partial f}{\partial \Lambda_{n}} \frac{\partial A_{n}}{\partial \lambda_{0}}=-\frac{\partial f}{\partial A_{n}} \cos q_{0} \cdot \sin \alpha ; \\
\frac{\partial f}{\partial q_{0}}=\frac{\partial f}{0 \Lambda_{n}} \frac{\partial 1}{\partial q_{0}}=-\frac{\partial f}{\partial \Lambda_{n}} \cos \alpha,
\end{gathered}
$$

dove a indica l'azimut della stazione considerata rispetto all'epicentro.

Indicando ron $(P n)$ i tempi di $P_{n}$ regi strati nelle stazioni di coordinate $q_{n}, \lambda_{n}$, quando si faceia astrazione degli inevitabili errori di osservazione e registrazione, detti tempi risulteranno della somma dell'ora iniziale del terremoto e del tempo di tragito corrispondente, per cui varrà l'equazione

$$
\begin{gathered}
\delta t_{0}-\cos \varphi_{0} \sin \alpha \frac{\partial f}{\partial \lambda_{n}} \delta \lambda_{0}-\cos \alpha \frac{\partial f}{0 \lambda_{n}} \delta \gamma_{0}+ \\
+\frac{\partial f}{\partial h_{n}} \delta h_{0}=\left(P_{n}\right)-t_{0}-f\left(\lambda_{0}, \varphi_{0}, h_{0}\right),
\end{gathered}
$$

che è l'equazione di conclizione relativa alla stazione considerata, di clistanza epicentrale $1_{n}$; il secondo membro è costituito da quantità note.

La [16] naturalmente va ripetuta per tutte le stazioni eal il sistema che cosi si ottiene risolto con il metodo dei minimi quallati.

5. - Numerosi sono i metodi per la determinazione dello spessore delle stratificazioni della crostan terrestre. Aleuni vemurero pure da me ideati, ma sa rebbe (roppo lungal la loro espositione: pertanto, rimando alle memorie origuinali $(1,20,36)$.

Ritengo peró utile richiamare ma formula rhe, fino dal $1936\left({ }^{2}\right)$, mi areva consentito di trovare la profondità approssimativa. a partire dalla quale la velocita delle onde $P n$ subisce un sensibile aumento; profonclità confermata suceessivamente da Beno Gutemberg.

Nel caso di terremoti che consentano chialre registrazioni di onde $P_{n}$ fino a distanze dell'ordine di $2000 \mathrm{Km}$, si ha modo di determinare nu valore applessimato dello spessore dello strato, nel quale le onde $P_{n}$ conservano velocità pressochè costante. Il punto in cui la dromocroma del'omda $P_{n}$ comincia ad inflettersi rerso l'asse delle distanze, compisponde sul tereneno alla distanza relativamente alla quale londa sismica comincia a penetrare in strati che consentomo maggiore velocità.

La plofondità approssimativa a cui viene a trovarsi questo strato può essere determinata quando si farecia l"ipotesi di ma densità crescente, in morlo miforme, con la profondità.

Se indichiamo con $e, n, r_{0}$ rispettivamente langolo d'emergenza del lagggio sismico in corrispondenza della superficie di Mohorovicice, la distanza angolare del punto origine (supposto in superficie) e il raggio terrestre, l'estensione di una teoria di Galitzin, consente di alrivare alla seguente formula, che serve al nostro scopo:

$$
d_{m}=\left[r_{0}-\left(d_{1}+d_{2}\right)\right]\left(1-\gamma^{\prime} o_{m}\right),
$$

dove:

$$
\varrho_{m}=1-\frac{\sin e \sin \frac{\theta}{2}}{\cos \left(e-\frac{\vartheta}{2}\right)}
$$

essendo $d_{m}$ fo spessore dello strato in cui le $P n$ hamno mal relocità sensibilmente costante. Il valore di $e$, e quindi di $\sin e$, puo esser dedotto con la notissima formula:

$$
v_{0}=V \cos e
$$

essendo $v_{0}$ la velocitì reale delle onde $P_{n}$ e $V$ lat velocità superficiale apparente delle onde $P_{n}$ per mat data distanza epicentrale. 
6. - Con i metodi aceemnati - e con altri rhe ho tralaciato - furono sturliati, da me e da alcuni miei collabomatori, numerosi terremoti, interessanti specialmente l'Europa centrale.

Per ció che riguarda la ristribuzione geografica delle scosse' esaminate dirò che esse riguardano il Veneto (in numero di $t)(1,2)$ l'alto Ardriatico (3) $\left({ }^{3}\right)$, la Valle Padana $(3)\left({ }^{11,38}\right)$, la Toscana $(4)\left({ }^{12,13,15,17,18}\right)$, il Lalzio (1), l'Abruzzo (2) $\left({ }^{19,21}\right)$, lo Jonio - preso le coste calabre $(1)\left({ }^{25}\right)$ - e presso le coste greche (1)( $\left.{ }^{17}\right)$-il basso Tirreno $(4)\left({ }^{18,22,23,24}\right) \mathrm{e}$ - fuori d'Italia - la Srizzera $(2)\left({ }^{10}\right)$, il Wurttemberg $(13)\left({ }^{10}\right)$, nonchè terremoti extra-europei, studiati nei loro riflessi sulla costituzione del continente europeo, come il terremoto del Turkestan del 2 novembre $1946\left({ }^{46}\right)$ e della dorsale mediana atlantica del 24 aprile $1947\left({ }^{38,30}\right)$. Nella fig. 5 sono riportate le posizioni epicentrali rella maggrior parte delle scosse esiminate.

Qui si vuole solo areennare some, dallo studio dei terremoti ritati, si siano potuti trarre alcuni elementi, atti a proporre con buona approssimazione - alcune ronclusioni sui valori delle relocità delle onde longitudinali e trasrersali, sulla stratificazione profonda del continente europeo, sulle radici delle Alpi e degli Appennini, sul meccanismo di produzione dei terremoti per cio che riguarda in particolare la zona relle Alpi, l'alto Arriatico e la zona degli Appennini.

Velocità di propagazione delle onde longitudinali.

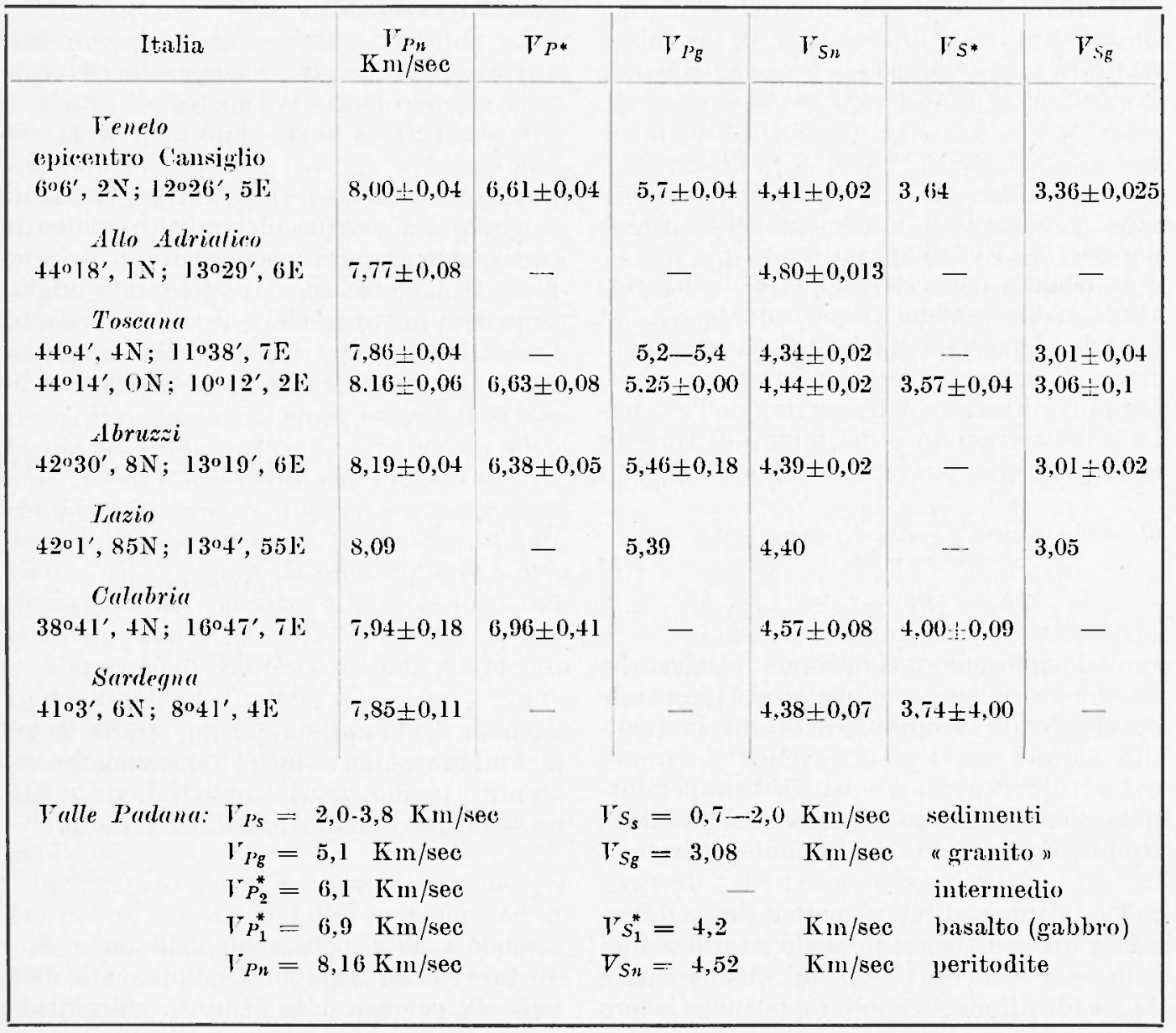


In un primo tempo sono stati rivelati, in modo chiaro, tre tipi distinti di onde longitudinali e trassersali, corrispondenti a tre diversi mezzi di propagazione: il mezzo ultrabasico, iniziale il mantello terrestre, il mezzo basaltico (o peroditico, o del gabbro) a il mezzo del "granito ».

Recentemente si è potuto rilevare, in corrispondenza della Valle del Po, l'esistenza di tre strati soverastanti, costituenti la crosta terrestre propriamente detta. Registrazioni di grosse esplosioni, aseguite nelle Alpi meridionali (zona del lago di S. Croce) hamno provato che lo stuato intermerlio si prolumga anche sotto le Alpi a ne costituise almeno nella parte meridionale - la parte preponderante (" ladice »).

Indicando con $P_{n}, P, P g$ le onde longitudinali interessanti i tre mezzi nell'ordine detto; con $s^{\prime \prime}, s^{*}, s_{g}$ le compispondenti onde trasversali, i valori delle corrispondenti velocità nei tre mezzi considerati figmano nella unita tabella.

Una ricerea sul terremoto del basso Tirreno del 16 marzo $1941\left(^{18}\right)$ ha messo in evidenza un noterole anticipo nei tempi di registrazione delle onde di dilatazione e di distorsione nelle stazioni più vicine all'epi(entro. Analoghe osservazioni sono state fatte nello studio del terremoto profondo delle isole Lipari del 13 aprile 1938, studio che as stato ripreso recentemente da Caloi e (Gioroi (22).

Altre ricerche condotte su teremoti interessanti la stessal zona, non hamno però confermato questa caratteristica, almeno lungo tragitti prossimi alla rerticale deggli ipocentri considerati.

Un'altä considererole anomalia che risulta dallosservaeione della tabella liportata consiste nell'elevato valore assunto dalla relocità delle onde trasversali destate dal terremoto dell'alto Arriatico del 30 norembre $1934\left(^{3}\right)$, in una zona calatterizzata da terremoti di sporofondamento. Un'analogal anomaliat es stata messa in evillenza da P. E. Valle per lo Jonio, dove, come per l'alto Adriatico, ad mal leggera diminuzione della velocità delle onde $P_{n}$ fa riscontro $n$ sensibile ammento della velocità delle onde $s$. Se indichiamo con $\lambda$ e $\mu$ le costanti di Lamé e con $g$ la densità del mezzo, nel tentativo di spiegare detta anomalia, P. E Valle condotto alla relazione (25)

$$
\frac{d \mu \mid}{u}<\frac{|d s|}{\varrho}<\frac{\mid d \lambda_{i}}{\lambda}
$$

che deve valepe atfinchè nel passare dal continente al Mediterraneo si osservi una diminuzione della relocità delle onde $P$ ' exd un aumento dellat relocita delle onde s'n.

sempre in tema di velocità di propagazione delle onde sismiche, in un ampio studio su diciassette teremoti rentro enroperi is stato possibile trovale che, nello strato di $20 \mathrm{Km}$ di "granito" — costituente la prima st ratificazione della crosta nell' Europa centrale - le onde longitudinali e trasversali dirette si propagano con relociti resesenti con la profondità, secondo le leggeri (12)

$$
\begin{aligned}
& T_{P g}=5,530(1+0.00158 \cdot h): \\
& r_{s_{g}}=3,275(1+0,00158 \cdot h)
\end{aligned}
$$

$p^{n+1}$

$$
\sigma=0,23 \text {. }
$$

7. - Stratifienziome della costa terrestre. Gli spessori degli strati, costituenti la cerosta terrestre, sono stati oftenuti con metodi basati o sulla propagazione delle oncle spaziali, o sulla dispersione delle onde superticiali, o sulla variazione del fattore

$$
k=\frac{v_{1} \cdot v_{2}}{v_{1}-v_{2}} .
$$

Le plime determinazioni riguardano lo spessore dello strato delle onde $I y$ in corrispondenza delle prealpi Carniche e del Cansiglio. Si sono ottenuti. in diverse determinazioni, valori variabili fra $40 \mathrm{Km}$ e 3t Km e si à concluso che lo spessore dello strato delle $P g$, in corrispondenza del sistema alpino olientale è di 35 K Km.co. Oggoi però, sulla base dei risultati formiti dallo studio di grosse esplosioni, si può concludere che tale spessore è costituito da "gubunito" solo neella parte superiore di $13 \mathrm{Km}$ ca.

Si è poi passati all'Italia rentrale e precisamente all'Aplemino toseo-romagnolo o alle Alpi Apuane. Lo spessore dello strato del "glanito" è risultato, rispettivamente, di $25 \mathrm{Km}$ a di $30 \mathrm{Km}$. In corrispondenza del Gran Sasso, determinazioni suceessive hammo portato ad un ralore di $25 \mathrm{~K}$ Kon, che sembra essere lo spessore medio del sistema degli Appennini. Altre ricerehe fecero ritenere che, 
in rorrispondenzal della Valle Padana lo spessore dello strato del "granito" sia alquanto minore (dellorrdine di $10 \mathrm{Km}$ ). La ronferma si is anta nello sturlio dei terresmoti della Val Pardana (1951), che hamno portato alla seguente suddivisione approssimativa dolla reosta terrestre:

$\begin{array}{lrc}\text { sedimenti } & 3-6 & \mathrm{Km} \\ \text { "granito" } & 10 & " \\ \text { basalto } & 7 & " \\ \text { gabbro (?) } & 11 & "\end{array}$

con la superficie di Mohorovicic alla profondità di 30-31 Km ("a. (fig. 4).

Nel 19:3 fu provalo, per la prima volta che, in ma vasta zona oltre il sistema alpino, comprendente almeno parte della Srizzera a il Württemberge $\left({ }^{10}\right)$, la prima superficie di discontinuta nell'interno della crosta terestere si troval ad una profondita di air'al

\section{8-20 KM}

valore suceressivamente confermato da altri ricereatori, quali Rothe, Potrschmit, Wannere, Reiche coullaboratori.

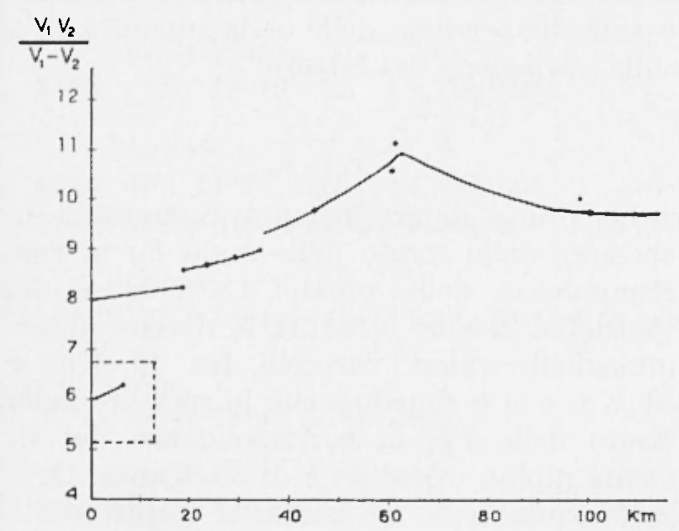

Fig. 2- Valore del cocelliciente $l=\frac{\ddot{c}_{1}=\ddot{c}_{2}}{v_{1}-v_{2}}$ in funzionc della jorofondjtio (Luropa centrale). 11 tratto nal riduadro si riferisce alla Val Padaua.

Il valore ottenuto nel 1943 provava ehe la superficie di dirontinuitì fra "granito" a basalto era. per la regione considerata, alquanto più sollevata di quanto nom fosse lino allora ritenuto.
Clteriori determinazioni. condotte con metodi basati sulla dispersione delle onde superficiali, hammo confermato per l'Italia un valore medio di $26 \mathrm{Km}$ per lo strato del "granito". Mentre tale strato, nella rlirezione" Jonio-Spagna (interessante gran parte del Mediterraneo) ha un valore medio di $15 \mathrm{~km}$ e nella direzione Jonio-Adriatico di $17 \mathrm{Km}\left({ }^{26}\right)$, lo spesssore dello strato del "granito nella direzione Turkestan-Roma è dell'ordine di 18 Ḱm $\left.{ }^{32}\right)$. Ai margini del continente, dal lato dell'Atlantico, tale strato sarebbe pressoches manchute $\left({ }^{38,39}\right)$.

Per quanto concerne la profondita della superficie di Mohorovicice, procedendo dalla Val Parlana verso $\mathrm{N}$ e $\mathrm{NW}$, si hamno i seguenti ralori approssimativi

$$
\text { Timeiren Autore }
$$

Val Padana $32 \quad$ Caloi e coll.

Alpi Meridionali tó Caloi

(Cansiglio)

Schwäbische Alb $34 \quad$ Caloi

Germania

Settentrionale 26-30 Reich, Schulze

Förtsch, Willmore

$\begin{aligned} \text { Atlantico } & 17 \quad \text { Caloi, Marcelli, } \\ & \text { Pannocchia }\end{aligned}$

Per l'Europa rentrale non è ancorar stato individuato lo strato intermedio. La sua esistenza però è provata dalla registrazione delle onde $C_{0,1}, C_{1,2}, C_{2,3}$ (originanti alla base della crosta e delle due discontinuità interne superiori, rispettivamente) ${ }^{37}$ ) in tutte le principali stazioni sismiche centroeuropee. Valgal per tutte un esempio tratto dalle stazioni di Kew e di Uccle. Ricerche successive dorramo - non ri è dubbio - provarne l'esistenza anche per altra via. [Fig. 3].

Natura delle scosse all'ipocentro e profondità ipocentrali. - Per quanto si riferisce al meccanismo di produzione dei terremoti studiati, va subito rletto che esso si è esplicato secondo tre diversi modelli: frattura (movimento del suolo dello stesso segno in quadranti opposti), sprofondamento (dilatazioni entro ma circonferenza di raggio determinato, compressioni all'esterno), solleva- 
Kew-Galitzin - E.W. $\Delta=8380 \mathrm{~km}$ (Aleutine 23-1.1935)
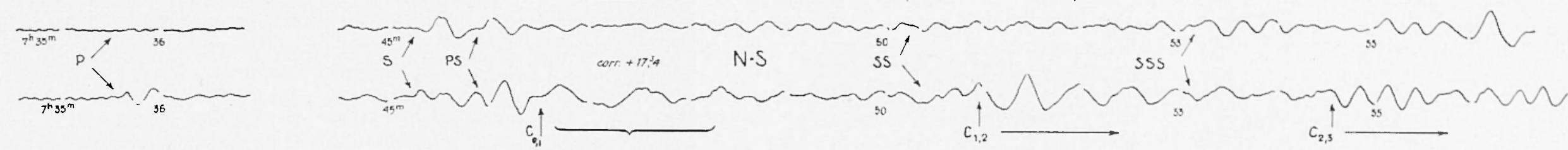

Uccle $\cdot$ Galitzin - E.W $\cdot\left(T_{0}=24,3 \cdot T_{1}=24^{3}, 5\right) \cdot \Delta=8500 \mathrm{~km}$ (Aleutine 25.1.1955)

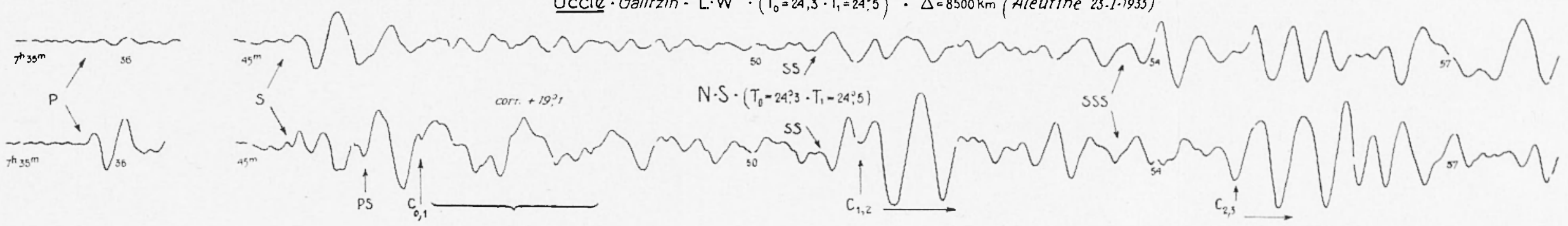

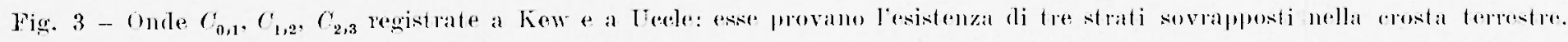



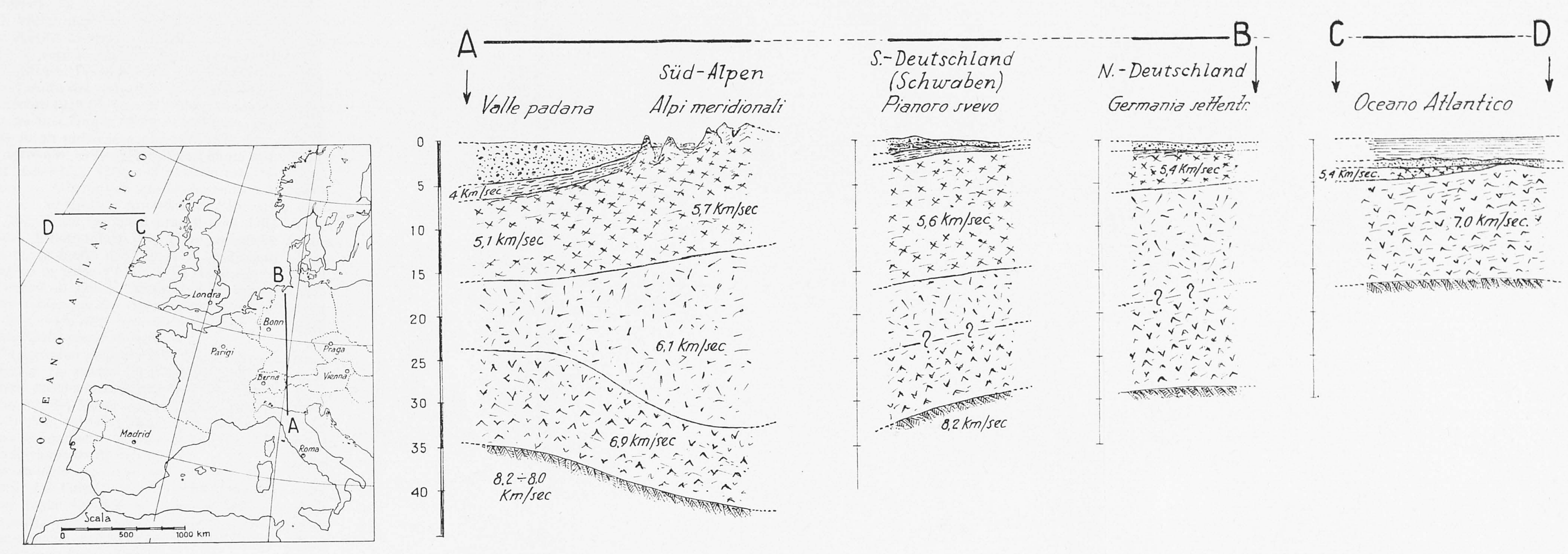

Alluvione

Terziario

Granito Bupertich di Mohorovicic 
mento (compressioni nell'interno di una ripconferenza di dato laggio, dilatazioni all'esterino).

Il primo modello (frattura con mutuo scorrimento di strati) è stato osservato in tutto il sistema alpino, dove esso sembra preclominarere: l'esempio più canatteristico è stato formito dal terremoto del Cansiglio del 18 oftobre 1936 ('), dovuto a frattula plovocata da una coppia di for"ze incelinate, giacente in un piano pule inclinato [Fig. 5].

I terremoti dellalto Adriation finora studiati presentano linvece il modello caratteristoco dei terremoti ali sporofondamento $\left({ }^{2,3}\right)$. I questo riguardo, i interessante osservare il parallelo nei fenomeni bradisismiei della l'egiones. E noto infatti ('he, in 's'e geologiches passate, fu molto ace(entuato il bradisismo negativo in tutta l'Istria e la Dalmazia: e dellentità di questo bradisismo si hamno chiare testimonianze (forma frastagliatissima dolla costa arlriatioa orientale, gran numero di isole presso le coste, exec.).

Lidpenentino presenta invere il fenomeno contrario. Lo studio dei termemoti dell'Appermino tosco-romagnolo (2,13), della Garfagnalla $\left({ }^{15}\right)$, del Monte Amiata $\left({ }^{16}\right)$ e, più l'ecentemente, deI Gran Sasso d'Itallia $\left({ }^{19}\right)$, ha messo chiaramente in luce il calattere di III sollevamento di strati.

La geologia concordal con queste constatazioni; fral l'altro, l'esame delle fronti di alcume faglie testimonia a favore dei sollevamenti, quali ad resempio quello del silente e quello dellax catema del Gran Sasso, verso Campo Imperatore.

Nel Tirreno meridionale i terremoti studiati rientrano invere nel modello caratteristico delle fratture. Qui però il femomeno presenta un interesse a sé, trattandosi di teremoti di origine profonda.

A proposito della profondità ipocentrale, va osservato che i terremoti interessanti il sistema alpino, in senso lato, riguardano esclusivamente la crosta terrestre: molti fol essi originano a piceole profondita ipocentrali. I)i 17 terremoti interessanti detta zona, 7 hamno avuto profondita inferiori ai $10 \mathrm{Km}$, 6 inferiori ai $20 \mathrm{Km}$ ed 1 solo ebbe origine alla base della cerosta teresestre $\left({ }^{10}\right)$.

I terremoti dellatto Arlriatieo sembrano originare da maggoiori profondità: probabilmente ai liniti della crosta terrestre.
Le scosse che arvengono nell'anbito degli Appennini originano invece, quasi escelusivamente, a piecolat profondità. inferiore ai 10 Kill.

MIi sembla di astremo interesise a questo riguardo, dare il dovuto risalto anche all'altro particolare che distingue la sismicità dell'Adriatico da quello della catema, ad esso parallela, degli $A p p e$ monini: l'opposto meceanismo da cui originano i terremoti nesle due zone contigue: agli sprofondamenti in profondita dellaltro Adriatico, fammo riscontro i sollevamenti superfieiali della catena appenninica. Sembla quasi che alle compressioni laterali determinate dai primi conseguano i solleramenti calusati dai serondi. A meno che le qualiliche di cantsal exlefletto non debbano exsere invertite. Ad ogni modo, siamo in presemza di un aspetto grandioso dellonogenesi ancol"a in at to nella regione in questione, come i confermato da molti altri indizi di carattere geologico.

Considerazioni a parte merital Ia sismicità del basso Timeno. Qui molti teremoti originano a grandi profondità quello del 16 mal'zo $19+1$ and una profondita di circa 85 Km ${ }^{18}$ ), quello delle isole Lipari del 13 aprile 1938 alla profondità di $300 \mathrm{~K}$ Km. (dirca (22), del basso Tirreno del 26 Dice. 1952 alla profondita di 265 Kin e delle isole Eolio del 23 Sovembre 195.1 profondo $2.10 \mathrm{Km} .{ }^{(21)}$.

La zona si presenta noterolnente anomala da tutti i punti di vista:geologicen, geodetico, sismieo. I Sord a a Sud della Sicilia, oltre che sull isola stessa, somo nulmerose le tracee di vulcanismo attivo o spento: lisola di Istica, d'origine vulcaniea. il gruppo insulare delle Lipari di recente origine vulcanica, eom i due vuleani attivi dello Stromboli a dell'isola di Vulcano, un vulcano spento trovasi a Sciale(a, Pantelleria c d'origine volucanica: tulta la zona insomma, senza citare i rulcani maggerori, è stata spesso teatro di aruzioni vulcaniche sottomarine, rivelate da teremoti a da maremoti.

Abbiamo aceemnato alla grande anomalia sulla propagazione delle onde sismiche provocate dai terremoti profondi citati a rivelata dal notevole antieipo nella legistrazione delle onde I'n ed s'n negli osservalori vicini alla zona epicentrale.

Poichè le onde trasrersali dammo deboli registrazioni nella zona, si è portati a rite- 


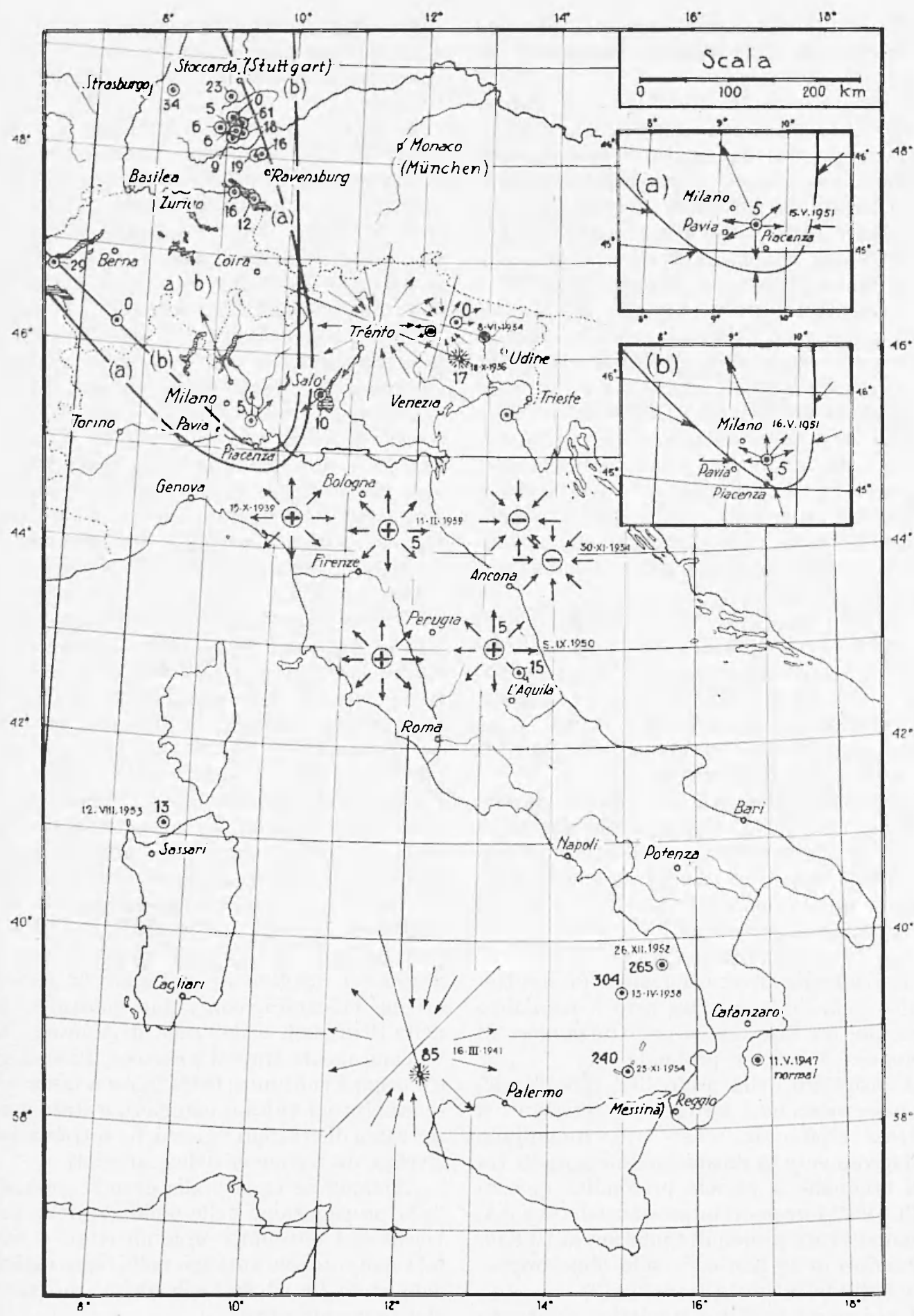

Fig. 5 - Posizione geografica di alcuni terremoti studiati in Italia dopo il 1933. la cifra in mrassetto indica la profondita ipocentrale. 
nere che le maggiori relocità non siano da attribuire ald una maggiore rigidita del mezzo, bensi ad un difetto di densitì da coollegarsi alle enormi riserve di materiale vul(anico. Un contributo in questo senso può dare pure la sottigliezza dello strato superficiale del "gl"anito" che, nella zona, sembra pressorchè mameante.

Strettamente collegata alla noterole anomalia sismica considerata, sta il fatto che la regione in parola è sede di mua cospicua anomalia glavimetrioa positiva.

8. - Al n. 5 ho riportato una formula che consente la determinazione della profondita. a partire dalla quale la velocita di propagazione delle onde $P_{n}$ comincia a crescere derisamente.

Ne ho fatto l'applicazione a due terre$\operatorname{moti}\left({ }^{2}\right)$.

Per il terremoto dell'alto Adriatico (30 Novembre 1934), ho ottenuto - riducendo al suo reale valore il tragitto pereorso dalle $P n$ al disotto della crosta terestre $-9=9^{\circ}$. Risulta inoltre $V=8.33 \mathrm{Km} / \mathrm{sec}$. Conseguono agevolmente i valori per e e $\mathrm{gm}$, e quindi

$$
d_{m}=90 \mathrm{Km} \text { (а). }
$$

Nello studio del terremoto del Cansionlio del $18 . \mathrm{X} .1936$ si c̀ ottenuto $)^{\circ}=10^{\circ}, \Gamma^{2}=$ $8,33 \mathrm{Km} / \mathrm{sec}^{2}$ Poirhè $v=8,00 \mathrm{Km} / \mathrm{sec}$, si ottengono i corrispondenti valori di e e quindi di $\varrho_{m}$. In definitiva

$$
d_{m}=85 \mathrm{Km}(\cdot \mathrm{a} .
$$

Fin dal 1935 avero quindi ottemuto che la velocitid delle onde longitudinali tende a crescere decisamente 85 -90 Km cirea al di sotto della superficie di Mohorovicice cioc ad una profondita di circa

\section{Kin.}

Come è noto, Gutenberg - circa la stessa epora - areva trovato che, per spiegame la zona dombla ("shadow zone "), era necessario ammettere l'esistenza di mo strato a bassa relocita (" low velocity layer n) alla profondita di $80 \mathrm{Km}$ (a). Pin recentemente, trovò che, in efletti, la relocità delle onde longitudinali decresce lievemente al disotto della crosra tererestere da 8.0 a $7.85 \mathrm{Km} / \mathrm{sec}$
(2) che tale minimo si trova fra 100 e $150 \mathrm{Km}$ di profonditì.

Di qui la seoperta degli strati e flessione di velocità e delle onde canalizzate Pa, Sa $\left({ }^{4+1}\right)$.

is eomunque interessante osservare ehe il valore sopra indicato di $120 \mathrm{Km}$, come profondita a partire dalla quale le velocità delle onde longitudinali tende a crescere derisamente, da me ottenuto nel 1935, hal avilto conferma nei recenti lavori compuinti da Gutenberg( $\left.{ }^{50}\right)$.

\section{RLASSUNT()}

Ina civea un cinquantemio si dibatte il complesso problema concernente le caratteristiche fisiche della crosta tervestre. Senza dubbio, sono stati compinti notevoli progressi da quando Mohorovicic provò l'esistenzu di due tipi di onde lomgitudimali (e trascersali); siamo però ancoin be" lungi dal ritonere risolto il problema.

Volta a colta, ricervatori isolati, o gruppi di studiosi, hamno portato il loro contributo all'auspicata risoluzione, recendo nuovi alementi di conoscenza. Qui si ruole accennare ai progressis conseguiti da italiani, negli ultimi renticinque anni.

Si riassumono, nella prima perte del lacoro, i metodi di calcolo di cui si é fatto maygjormente uso. Aleuni di questi metodi sono stati ideati allo scopo di vidure al minimo gli crori devicunti da imperfetta conoscenza dei tempi di arrico delle varie fasi o, addirittura. di prescindere da eventuali incertezze nella correzione dei tempi. Si i inoltre ritenuto opportumo striluppare metodi di calcolo, nei quali le relociti di mopugnaione entrassero come incogmite.

si riportano quindi alenni dei risultati oftemuti, con particolare riguardo alle .1lpi centro-meridiomali, agli Llppemini, alla Talle padena e ad una rasta zona del ll wrttembery.

Lo struto del "graniton" consente be velocitie piic elecute per le onde P' $\mathrm{g}$, in corrispondenza delle Alpi $(5, \overline{5}-5,8 \mathrm{Km} / \mathrm{sec})$, mentre si osserea un minimo in corrispondenza della Valle del Po e della Germania settentrionale (5, 1 I $\mathrm{m}$ iseci).

Lo spessone di detto strato passa dai $6 \mathrm{Km}$ della Germania Settentrionale, ai 10 della Talle Padama, ai 13 dolle 1 1lpi Meridionali, 
ai 18 del Wurttemberg, per giungere ai 25 ca. dell 1 puemino centro-settentrionale.

Le stratificazioni successive, costituenti la rosta tervestre, conducono onde longitudinali con velocitì non ancora ben precisate; in media, esse risultano dell'ordine di $6,45 \mathrm{Km} / \mathrm{sec}$ (onde $\left.P^{*}\right)$, variando da 6,1 a $7,0 \mathrm{Km} / \mathrm{sec}$. Tali veriazioni somo, con ogni probabilita, da attribuire all'esistenza di almeno due strati al di sotto di quello del "granito ": lo strato intermedio (basalto) - com velocitic dell'ordine di $6,1 \mathrm{Km} / \mathrm{sec}$ per le onde longitudinali - lo struto del gabbro? con velocità per le onde longitudimali dell'ordine di $\mathrm{T}, 0 \mathrm{Km}$ sec. L'esistenza di tre strati socrapposti $\grave{e}$ del resto provata dalla registrazione, in tutte le stazioni sismiche europee, di onde del tipo $C_{0,1}, C_{1,1}$, $\mathrm{C}_{2,1}$, mendenti origine alla base della crosta terrestie, e alle due discontinuitri piu prossime alla superficie esterua, rispettivamente.

Lo spessore complessivo della crosta terrestre, si mostra massimo sotto i sistemi montuosi (50-55 Km per gli Appennini centrali; 40-45 Km per le Alpi meridionali).

La "rallice " delle Alpi meridionali (Cansiglio), sembra formata dallo strato intermedio. In corrispondenza della Valle padana e del Wurttemberg non sembra superare di molto $i$ $30 \mathrm{Km}$, mentre nel bacino atlantico si mostra dellordine di $1 \mathrm{i}$ Km ca.

Lo strato det "granito" si viduce notevol. mente di spessore in corrispondenza dei grandi mari; manca forse in gran pate del Tirreno meridionale, cosi come al largo dello scoglio di Rockall, nell'Atlantico settentrionale.

$I$ sedimenti - sciolti e consolidati - hamo spessori notevoli nella Falle padana, variando dai 3 ai $7 \mathrm{Km}$ in media.

Per quanto riguarda la parte superiore del "mantello ", va ancora osservato che, gia dal 1936, era stato possibile all A utove di provare che la velociti di propagazione delle onde $\mathrm{Pn}$, tende decisamente arl anmentare a partive da $120 \mathrm{Km}$ di profonditi cirea. Com'è noto, questa mofonditi costituisce, per cosi dire, l'asse dell'astenosfera, dove massima è la flessione della velociti delle onde longitudinali.

\section{ABSTRACT}

The complex problem of the physical features of the earth's crust has been debated for about fifty years. There is no doubt that since the day when Mohorovicic demonstrated the existence of two types of longitudinal (and transremsal) waves, considerable progress has been achieverl. The are, nevertheless, still remote from considering that this problem has. been solved.

From time to time, individual researchers or groups of scientists have given their contributions towards the desired solution, bringing fresh data to our linowledge. Here, we should like to indirate the progresss achieved by Italian savonts during the past twenty-five years.

The first part of our worl summerizes the methods of calculation which have been used mostly. Some of these methods have been worked out in order to reduce to a minimum the errors deriving from our imperfect linowledlye of the times of the arrival of various stages or even in order to malie us independent from the eventual unertainties in the correction of times. It has been exen considered advisable to work out methods of calculation in which the speed of propagation is included as an unlinowen quantity.

Next, some of the results obtained are quoted, with particular regard to Central-Southern Alps, Apennines, Valley of the Po, and a large part of Wurttemberg.

The layer of "granite " allowes greater speeds for $P$ a waves, in correspondence with the Alps (5.7 or 5.8 Kilometres per second) while the minimum velocity is observed in correspondence with the Valley of the Po and in Northern German!y (5.1 litometres per second).

The thicliness of this layer reaches 6 kilometres in Northern Germany, 10 in the Falley of the Po, 13 in the Southern Alps, 18 in Tirttemberg, and as in Central-Northern $A$ peminines.

The successive strata of which the crust of the earth consists conduct longitudinal waves with velocities that have not been ascertained accurately. Generally spealing, they acerage something lile 6.45 litometres per second ( $P^{*}$ waves) varying from 6.1 to 7 lillometres per second. These variations are to be attributed, in all probabitity, to the existenee of at least two layers beneath the "granite" i.e. the intermediate stratum (basalto) carrying longitudinal waves at about 6.1 litometres per sec. and a stratum of gabbro, in which longitudinal naves travel at the velocity of about $i$ litometres per second. In fact, the existence of 
three superimposed strata has been de monstrated at all European seismic stations by recording of waves of $C_{0.1} C_{1.1}, C_{2.1}$ type, originating respectively at the base of the earth's crust and at the two discontinuities that are the nearest to the external surface.

The aggregate thicliness of the earth's crust appears to be greater beneath mountrin ranges (50) to 55 kilometres in the Central Apennines and 40 to 45 litometres in the southern Alps).

The "root no the Southern Alyss (Cansiglio) seems to consist of the intermediary stratum. In the Valley of the Po and in Wrirtemberg it does not seem to exceed 30 litometres much, while in the Atlantic basin it appears to be about 17 lilometres thick.

The thickness of "granite" diminishes considerably beneath the great seas. It is probably absent beneath most of the Southern Tyrrhenian in a manner similar to that met with off the Rockall reef in Northern Atlantic.

Sediments, loose and consolidated, are endowed with considerable thickness in the Talley of the Po, ranging as they do, from 3 to 7 litometres on the average.

As regard the upper part of the "mantle", it should be also observed that already since 1956, the Author has been able to prove that the velocity of the propagation of $P_{n}$ waves tends decidebly to increase, beginning with the depth of about 120 kilometres. As noted, this depth constitutes, so to speal, the axis of the astenosphere, where the flection of the velocity of the longitudinal waves is at its maximum.

\section{BIBLIOG R.IFIA}

(1) CanoI, P., Rirevehe su lerremoli ad origine vicina. Scosse del Cansiglio dellollobre 1936, "La Ricerea Scientifica ", IX, (1938).

$\left({ }^{2}\right)$ - Allivild Sismica in Ilalia nel decenmio 19.30-19.39, Le Monnier, Firenze, 1942.

$\left(^{3}\right)$ - Il terremolo adrialico del 30 Novembre 19.34 "Boll. Soe. Fism. It.", XXXV. (1937).

${ }^{(1)}$ - Nuovi melodi per la delerminaziome delle coordinale epicentrali e della profondila ipocentrale di un terremoto ad origine ricina, "La Ricerca Scientifica ", X, (1939).

$\left.{ }^{5}\right)$ - Sopra un nuovo metodo per calcolare le profondilà ipocentrali, "I La Ricerca scien. tifical », XI, (1940). $\left.{ }^{6}\right)$-- Sulla delerminasione delle coomdinate spasiali di un lerremolo ad origine vicina. "Boll. soc. Sis. It. ", XXXVIII. (1940).

(') - Calcolo delle profondià ipoesulrali in junzione della dislamsa epicentrale e dellangolo demergensa delle onde $P \eta$. "La Ricerea Scient.", V. (1934).

${ }^{8}$ - Delerminazione delle coordinale ipocenbali di un terremolo ad origine ricina con i lempi delle onde longiludinalie trascersali direlle, "I La Riearea soient." XII, $(19+1)$.

(9) --. Suovo melodo per delerminare le coordinale ipocentrali e le velocili di propagasione delle onde longiludinali e resiversali direlle. "Rent. R. Aec. d'Italia", serie VII, IV, (19+3).

$\left({ }^{10}\right)$ - Caralleristiche sismiche fondamentali dell Europa Cembrale, quali risullamo dallo sludio di 17 lerremoli eenlro-enropei ", "Boll. Soc. Sis, Ital.", XI, (1942).

(11) - Epicentro e profonditi ipocentrale del terremolo di Sali del 2t Gemmaio 1943, "Istituto Veneto di scien., Lett. al Arti ", CIV. (19t+-45).

$\left.{ }^{12}\right)$ - Il lememolo dell Appenmino loseo-roma quolo dell 11 Febbraio 1939, "La Ricerea sucient. ", X. (1939).

${ }^{\left({ }^{3}\right)}$ - Caralleristiche sismiche dell Appennimo loseo-romagnolo, "I La Ricerea scient.". XI, $(1940)$.

${ }^{(14)}$-- Sulla delerminazione delle coordinale epicenbali di un lememolo ad origine $v i$ cina, "Atti Ace. clelle Scienze di Torinon. 81 e 82, (1945-46, 1946-47).

(15) Rosixi, F.. Il terremolo della Garfagnama del 150llobre 1939, "l la Ricercal šcient." XI. $(1940)$.

(16) D) Finippo, 1)., $I 1$ terremolo del Monle Amiala del 19 Gingno 19.40, "Ia Ricerca scient.". XII, (19+1).

(17) Giorai, M.. 17 lememolo del Momle Amiala del 16 Ollobre 1940. "I La Ricercal Scient.", XII. $(19+1)$.

(18) I)] FuldPPo, I)., Sludio microsismico del terremolo del basso Tirremo del 16 Marao $19+1$, "Boll. Soc. Sis. lt.", XXXIX. $(19+1)$.

(19) - e Marceldi, L., Cno s/udio del terremolo del Gran Sasio dilatia del j sellembre 1950, "Annali di (ieofisican, IV. (1951).

(20) CAlor. P.. Sulla velocili di mopagaziome delle onde $P^{*}$ e sullo spessore dello stralo del qramilo well'Europa reulrale, "I La Ricerca scient. s, XI, (1940).

(21) I)I Filuppo. I), Marcenis, Ja, Tempi di brafillo delle onde $P^{*}$ e spessore dello strato del gramilo nellitalia Genlrale, "Annali di Geofisica", IV, (1951). 
(22) Canor, P., Girorai, M., Sludio del lervemoto delle isole Lipuri del 1.3 iprile 19.38, "Anuali di Geofisica", IV, (1951).

(23) GIrLaxisa, A., Sludio di un movimento sismico del basso Tirreno, originanle alla profondili di $265 \mathrm{i} \mathrm{h}$. "Rend. Acc. Naz. dei Isincei ", XVIII, (1955).

(21) .... Sul terremolo profondo delle isole Eolie del 2.3 Novembre 19.5.t ". "Anmali di Geofisica ", VIII, (1955).

${ }^{\left({ }^{25}\right)}$ Vat.e, 1'. E., Contributo allo sludio delle caratteristiche sismiche del Medilerraneo centro-orientale, "Ammali di Geofisica", I, (1948).

$\left({ }^{26}\right)$ Festa, C., VAlse, P. F., Cua valutazione dello spessore dello slralo del "granilo" nel Mediterraneo centro-orienlale, "Annali (li Geofisica », I, (1948).

$\left.{ }^{27}\right)$ Rotiĺ, J. P., Peterscinate, H., Elude séismique des explosions d Haslach, "Ann. lnst. Pliys. Globe ", V, 13, (1950).

(28) Reicin, H., Scinuze, G. A., Fortscil, 0., Das geophysikalische Ergebnis der Sprenqung von II aslach im sudlichen Schararwald, "Geol. Rundschau", XXXVI, (1948).

$\left({ }^{29}\right)$ - Uber seismische Beobachtungen der PRATLA von heflescionen aus grossen Tiejen bei den grossen Sleinbruch-sipre"gungen in Blaubeuren am t. März und am 10. Mai 19:2, "Geol. Jahrb", B.68, llamnover, 1953.

(30) Gatai, J., S'Tegena, I.., Nagymélységü reRexiok Majduszoszolo vidélén, "Geofiz. Közlem », 4, Budapest, 1955.

(31) Gayburcev, G. A., Veizuan, P. S., TuIINA, I. V., La strulura della crosta lerrestre nella regione del Tian-Scian settentrionale, secondo $i$ dali del sondaggio sismico di projondili, "Ace. delle sicienze URSS", 105, 11. 1, (1955).

(32) Peronaci, F., Delerminazione del valore medio dello spessore dello strato di granito per i ragilli Turkestan-Europa, "Annali di Geofisica ", III, 4, (1950).

${ }^{(33)}$ Winmore, P. I., Seismic erperimenls on the Norll German explosion, 19t6 to 19.4, "Phil. Trans. R. Soc.", A, CCXLII, (1949).

(21) Sicmulze, G. A., For'trcir, O., Die seismischen Beobachlungen bei der sprengung auf IIelgoland am 18. April 19.5 ₹ur Erjorschung des tieferen Unlergrundes, "Geol. Jahrlı.", B. 64, Ilannover, 1950.
("5) Reich, H., Fortscit, O., Sciudzze, G. A. Results of seismic observations in Ger. many on the Heligoland explosion of April I8, $19 \pm \%$.

(30) Canoi, P., De Pantilis, M., Di Finiplo, D., ILARCElli, I., SPADEA, M. C.. Terremoti della Tal Padana del I.j-16 Maggio 19:51, "Anmali di Geofisica", IX, (1956).

$\left({ }^{37}\right)-Q_{i, j}$, "Anmali di Goofisica", VIII, (1955).

$\left.{ }^{38}\right)$ - Marcelit, I.. Pañoccila, G., Ancora sulla velociti di propagazione delle onde superficiali per tragitti suballantici, "Annali di Geofisica ». III, 2, (1950).

$\left(^{39}\right)$ - Marcenti, L., Onde superficiali allraverso il bacino dell Allantico, "Annali di Geofisica ", V, 3, (1952).

$\left.{ }^{10}\right)$ - Comporlamento delle onde di Rayleigh in un mezzo firmo-viscoso indefnito, "Annali di Geofisican, I, (1948).

${ }^{(11)}$ - Teoria delle onde di Rayleigh in mezzi elaslici e firmo-elastici, esposta con le omografie vettoriali, "Archiv für Met., Geopl. und Biokl. ", IV, (195I).

${ }^{(2)}$ Kubotera. Akira, Rheological Properties of the Earth's Crust..., "Journal of P'hysics of the Eartl s, I, 1, (1952).

(i3) Canor, P., Sulla dispersione delle onde si. smiche nell ambilo delle altissime frequen. ze, "Atti Ace. Naz. dei Lincei", serie VIII, Marzo 1958

${ }^{(4)}$ - Onde longitudinali e trasversali guidate dallaslenosfera, "Renıl Acc. Naz. dei Jincei ", serie VIII. XV, (1953).

${ }^{\left({ }^{5}\right)}$ - L'aslenosfera come camale-guida della energia sismica, "Anmali di Geofisica", VII, (1954).

(16) - Peronaci, F., Il terremoto del Turlieslan del 2 Novembre 19-4\%, "Anmali di Geofisica " I, (1948)

(17) Di Finiepo, D., Marceria, I.. Uno studio sul lerremolo di Cefalonia del 1: Lyosto $1953 . .$. , "Anmali di Geofisica", VII. (1954).

$\left.{ }^{(4)}\right)$ REICI, II.. In. Suddeutschland seismisch ermittelle Tiefe Grensflichen und ihre geologische Bedeulung, "Geol. Rundschau ", B. 46. (1957).

(19) Brockimp, B., Seismische Beobachtungen bei sleinbruchsprengungen, "Zeitschrift für Geophysik (1931).

${ }^{50}$ - Zum Werdegang der Torliejen, "Geologie ", IV, (1955).

(51) Gutenibers, B., Wave Telocities al Denths Between 50 and to0 hilometers. "Bull. Seism. Soe. Am. ", 43. (1958). 